\title{
Análise quantitativa das zeólitas ferrierita, ZSM-5 e mordenita presentes em amostras sintetizadas
}

\section{(Quantitative analysis of zeolite ferrierite, ZSM-5 and mordenite present in synthesized samples)}

\author{
B. J. B. Silva, A. O. S. Silva, P. P. M. Neto, T. P. M. Costa, L. V. S. Júnior, L. M. O. Ribeiro, \\ S. L. Alencar, A. E. Silva, R. B. Santos \\ Universidade Federal de Alagoas - UFAL, Campus A. C. Simões, Av. Lourival de Melo Mota, s/n, \\ Tabuleiro dos Martins, Maceió, AL 57072-900 \\ brunojbarros@hotmail.com
}

\begin{abstract}
Resumo
Nos estudos de obtenção de materiais zeolíticos é comum a formação de misturas de fases cristalinas devido à estabilidade relativa destes materiais no meio reacional empregado na síntese. Desta forma, é extremamente importante quantificar as diversas fases presentes no sistema para otimizar os métodos de sínteses de uma determinada zeólita sem a presença de contaminantes. Especificamente, foi verificado que durante a síntese das zeólitas ZSM-5(MFI), mordenita (MOR) e ferrierita (FER), em diversas condições experimentais, ocorreram a formação de misturas de fases binárias (FER+MOR) e (FER+MFI), às vezes ternárias $(\mathrm{FER}+\mathrm{MOR}+\mathrm{MFI})$. O propósito do trabalho foi a realização de várias misturas binárias em diversos percentuais destes materiais, a fim de se obter uma metodologia para quantificar a presença de cada uma das fases (FER, MOR e MFI) em amostras sintetizadas sob diferentes condições experimentais. Com este objetivo, foram preparadas misturas físicas com diversos teores das fases MFI, MOR e FER, a partir de amostras altamente cristalinas de cada fase. Estas fases de composições conhecidas foram analisadas por difração de raios X para quantificar as áreas dos picos característicos de cada material, podendo-se assim obter uma relação de percentual de fase/área de cada componente da mistura.
\end{abstract}

Palavras-chave: difração de raios X, zeólitas.

\begin{abstract}
In studies for obtaining zeolitic materials common to the formation of mixtures of crystalline phases due to the relative stability of these materials in the medium used in the synthesis. Thus, it is extremely important to quantify the various phases present in the system to optimize methods of synthesis of a zeolite without determining the presence of contaminants. Specifically, it was found that during the synthesis of zeolites ZSM-5(MFI), mordenite (MOR) and ferrierite (FER) in various experimental conditions, is the formation of binary mixing phases (FER + MOR) and (FER + MFI), sometimes ternary (FER + MOR + MFI). The purpose of this work was to perform various binary mixtures at various percentages of these materials in order to obtain a methodology to quantify the presence of each of the stages (FER, MOR and MFI) samples synthesized under different experimental conditions. With this objective, physical mixtures were prepared with different contents of the phases MFI, MOR and FER, from highly crystalline samples of each phase. These known compositions phases were analyzed by X-ray diffraction to quantify the areas of the peaks characteristic of each material, thus being able to obtain a ratio of the percentage of phase/area of each blend component.
\end{abstract}

Keywords: X-ray diffraction, zeolites.

\section{INTRODUÇÃO}

As zeólitas são aluminossilicatos cristalinos contendo canais microporosos e/ou cavidades em sua estrutura. Sua rede cristalina é constituída de tetraedros de $\mathrm{SiO}_{4}$ e $\mathrm{AlO}_{4}$ ligados por átomos de oxigênio, formando assim uma rede tridimensional de tetraedros $\mathrm{TO}_{4}[1]$. O estudo destes materiais desperta cada vez maior importância devido algumas de suas propriedades intrínsecas (estabilidade térmica, acidez, entre outras), e também devido a algumas de suas aplicações como trocadores iônicos, adsorventes e catalisadores na indústria petroquímica [2].

A técnica de difração de raios X (DRX) é a mais indicada na determinação de fases cristalinas, pois na maioria dos sólidos (cristais), os átomos se ordenam em planos cristalinos separados por distâncias da mesma ordem de grandeza dos comprimentos de onda dos raios X. Dentre as principais vantagens da técnica, destacam-se a simplicidade e rapidez do método, a confiabilidade dos resultados e possibilidade de análise quantitativa destas fases [3]. A difração de raios $\mathrm{X}$ pelo método do pó é uma ferramenta essencial para a identificação e caracterização das zeólitas em vários estágios de sua síntese, permitindo a identificação e quantificação das fases cristalinas, determinação de parâmetros cristalográficos e tão como características físicas e morfológicas [4]. Ás vezes através da comparação entre os difratogramas de 
materiais em estudo com os padrões da literatura observase que as posições dos picos são idênticas, mas suas intensidades relativas não estão de acordo com as do material padrão. As possíveis causas são: remoção do direcionador orgânico dos poros, mudança do cátion de compensação (troca iônica), orientação preferencial e substituição de íons dentro da estrutura cristalina (substituição isomórfica) [5]. O percentual de cristalinidade é determinado através da soma das áreas dos picos escolhidos para o material em análise, dividido pela soma das áreas dos mesmos picos da amostra padrão, a qual é assumida ser $100 \%$ cristalina, conforme a equação $\mathrm{A}[6]$.

$\%$ Cristalinidade $=\frac{\sum \text { Áreas dos picos (amostra) }}{\sum \text { Áreas dos picos (padrão) }}$

As zeólitas e a análise por DRX estão intimamente associados desde o início do estudo destes materiais, onde através da qual foram identificadas diversas novas estruturas [7]. O processo de síntese das zeólitas é uma tarefa que envolve certo grau de dificuldade e que pode tornar-se extremamente exigente, principalmente quando se requer um material com uma determinada estrutura e composição química, com elevado grau de pureza (sem fase amorfa ou impurezas cristalinas referentes a outras estruturas), existindo diversos parâmetros que influenciam a obtenção da estrutura desejada (composição do meio reacional, temperatura, tempo, entre outros) [8]. A análise por DRX é uma técnica que oferece a possibilidade de determinar a estrutura de zeólitas e outras mais complexas, tão como a análise quantitativa de fases em misturas [9], sendo esta de suma importância para a compreensão molecular detalhada destas estruturas e de suas propriedades [10].

Este trabalho teve como proposta a realização de misturas binárias (ZSM-5/ferrierita e mordenita/ferrierita) em diversos percentuais (10-95\%), a fim de se obter uma metodologia para quantificar a presença de cada fase nas amostras de zeólitas sintetizadas, pois estas são motivo de estudo devido ao grande interesse industrial, por exibir notável desempenho catalítico e uma variedade de reações tipo redox e ácidas como: isomerização de n-alcanos em isoalcanos, craqueamento de n-parafinas, isomerização do m-xileno, o-pineno, diclorobenzenos, dentre outras.

\section{EXPERIMENTAL}

\section{Síntese da zeólita ferrierita (FER)}

A síntese da FER foi realizada de acordo com método descrito por Jacobs e Martens (1987) [11], utilizando-se a seguinte composição de gel: $1,85 \quad \mathrm{Na}_{2} \mathrm{O}: \mathrm{Al}_{2} \mathrm{O}_{3}: 15,2 \quad \mathrm{SiO}_{2}$ :5,92 $\mathrm{H}_{2} \mathrm{O}: 19,7 \mathrm{C}_{2} \mathrm{DN}$, com hidróxido de sódio (Merck) como fonte de sódio, aluminato de sódio (Sigma Aldrich) de alumínio, sílica coloidal (Alfa Aesar) de silício, etilenodiamina ( $\mathrm{C}_{2} \mathrm{DN}$, Merck) como direcionador de estrutura, e água destilada como solvente. O procedimento de síntese ocorreu nas seguintes etapas: dissolução sob agitação do aluminato de sódio e hidróxido de sódio em água, dissolução da sílica sol com a etilenodiamina, mistura das soluções (I) e (II) até completa homogeneização. Após a preparação do gel, este foi transferido para um vaso de teflon revestido com uma autoclave de aço inoxidável e aquecido a $177{ }^{\circ} \mathrm{C}$ por $240 \mathrm{~h}$. Após a cristalização, a autoclave foi retirada da estufa e resfriada até temperatura ambiente. $\mathrm{O}$ seu conteúdo foi transferido para um becker contendo $100 \mathrm{~mL}$ de água destilada, sendo em seguida submetido a um banho de ultra-som por 25 min para homogeneização das partículas formadas. Finalmente, o sólido resultante do processo de cristalização foi separado do líquido sobrenadante por filtração a vácuo, lavado diversas vezes com água destilada (até o pH do filtrado atingir o valor de 7) e seco em estufa a $100{ }^{\circ} \mathrm{C}$ por $12 \mathrm{~h}$.

\section{Síntese da zeólita ZSM-5(MFI)}

A amostra de ZSM-5 foi sintetizada com sílica amorfa (Merck) como fonte de silício, hidróxido de sódio (Merck) como fonte de sódio, sulfato de alumínio octadecahidratado - $\mathrm{Al}_{2}\left(\mathrm{SO}_{4}\right)_{3} \cdot 18 \mathrm{H}_{2} \mathrm{O}$ (Merck) como fonte de alumínio, brometo de tretapropilamônio (TPABr) como direcionador estrutural (Merck) e água destilada como solvente [12]. Os materiais precursores foram misturados em proporções estequiométricas para se obter um gel com a seguinte composição molar: 10,6 TPABr :14,3 $\quad \mathrm{Na}_{2} \mathrm{O}: 2,0 \quad \mathrm{Al}_{2} \mathrm{O}_{3}$ : $100 \mathrm{SiO}_{2}: 2000 \mathrm{H}_{2} \mathrm{O}$. O procedimento para a obtenção do gel para a síntese da ZSM-5 consiste nas seguintes etapas: dissolução do hidróxido de sódio em metade da água requerida na síntese, adição da sílica gel seguida de forte agitação por $1 \mathrm{~h}$ (solução A), dissolução do brometo de tetrapropilamônio no restante da água, adição do sulfato de alumínio octadecahidratado seguida de agitação por 1 h (solução B), mistura das soluções A e B mantendo-se o sistema sob agitação por mais $2 \mathrm{~h}$. Após a obtenção do gel de síntese, este foi transferido para um vaso de teflon revestido com uma autoclave de aço inoxidável e aquecido a $150{ }^{\circ} \mathrm{C}$ por $168 \mathrm{~h}$. Após acristalização, o sólido foi recuperado pelo procedimento similar ao utilizado para a zeólita ferrierita e seco em estufa a $100^{\circ} \mathrm{C}$ por $12 \mathrm{~h}$.

\section{Sintese da mordenita}

A síntese da mordenita foi efetuada utilizando-se como reagentes precursores: silicato de sódio (Pernambuco Química) como fonte de sílica, hidróxido de sódio (Merck) como fonte de sódio, sulfato de alumínio octadecahidratado (Merck) como fonte de alumínio, e água destilada como solvente. Os materiais precursores foram adicionados em proporções estequiométricas de modo a se obter um gel com a seguinte composição molar: $9,02 \mathrm{Na}_{2} \mathrm{O}: 1,00 \mathrm{Al}_{2} \mathrm{O}_{3}$ : $20 \mathrm{SiO}_{2}: 300 \mathrm{H}_{2} \mathrm{O}$. O procedimento para a obtenção do gel para a síntese da mordenita consiste das seguintes etapas: dissolução do hidróxido de sódio em um terço da água requerida para a síntese (solução $\mathrm{A}$ ), dissolução do sulfato de alumínio em um terço da água requerida para a síntese 
(solução B), dissolução do silicato de sódio em um terço da água requerida para a síntese (solução $\mathrm{C}$ ), mistura das soluções A, B e C mantendo-se o sistema sob agitação por $1 \mathrm{~h}$. Após a obtenção do gel de síntese, este foi transferido para um vaso de teflon revestido com uma autoclave de aço inoxidável e aquecido a $170{ }^{\circ} \mathrm{C}$ por $72 \mathrm{~h}$. Após a cristalização, o sólido foi recuperado pelo procedimento similar ao utilizado para a zeólita ferrierita e seco em estufa a $100{ }^{\circ} \mathrm{C}$ por $12 \mathrm{~h}$.

\section{Análises térmicas (TG/ATD)}

As zeólitas (FER, MOR e ZSM-5) foram submetidas a análises de TG/ATD, para a obtenção das quantidades de água intracristalina (umidade) ocluída. Estas foram realizadas numa termobalança Shimadzu DTG $60 \mathrm{H}$, com uma taxa de aquecimento $10^{\circ} \mathrm{C} \mathrm{min}^{-1}$ na faixa de temperatura de ambiente até $500^{\circ} \mathrm{C}$ por $1 \mathrm{~h}$; após, com a mesma rampa de aquecimento o material foi aquecido até $800{ }^{\circ} \mathrm{C}$ por $1 \mathrm{~h} \mathrm{em}$ atmosfera dinâmica de nitrogênio, na vazão de $50 \mathrm{~mL} \cdot \mathrm{min}^{-1}$, em cadinhos de alumina de $70 \mu \mathrm{L}$ e uma massa $\sim 10 \mathrm{mg}$.

\section{Misturas}

As misturas foram feitas em diversas proporções (1095\%) para massa de $1 \mathrm{~g}$ de amostra (massas corrigidas devido ao percentual de umidade de cada material), onde estas foram maceradas num almofariz com pistilo, e após, colocadas num micromoinho IKA Ultra Turrax, por $30 \mathrm{~min}$ cada, para homogeinização.

Sínteses das amostras binárias (ferrierita+ZSM-5 e ferrierita+mordenita) e ternárias (ferrierita+ZSM$5+$ mordenita)

Para se obter amostras contendo misturas binárias e ternárias das zeólitas ferrierita (FER), ZSM-5 e mordenita foram realizados experimentos de síntese com diversas condições experimentais. Os parâmetros, razão sílica-alumina (SAR), alcalinidade do meio $\left(\mathrm{OH} / \mathrm{SiO}_{2}\right)$, concentração de direcionador orgânico adicionado, concentração de água no gel, fontes de silício, alumínio e sódio, entre outros. A partir deste amplo conjunto de experimentos, foram selecionadas as condições experimentais que geraram amostras isentas de fases amorfas, contendo misturas de fases binárias e ternárias das zeólitas mordenita, ZSM-5 e ferrierita. Estas condições experimentais são definidas, quase sempre, em termos da razão $\mathrm{SiO}_{2} / \mathrm{Al}_{2} \mathrm{O}_{3}$ (SAR) e da alcalinidade do meio (razão molar $\mathrm{OH} / \mathrm{SiO}_{2}$ ). As composições das misturas reacionais usadas nas sínteses estão agrupadas por SAR e com as variações na razão $\mathrm{OH} / \mathrm{SiO}_{2}$, conforme indicadas na Tabela I.

Nas sínteses das amostras foram utilizados os seguintes reagentes precursores: hidróxido de sódio (Sigma Aldrich) como fonte de sódio, aluminato de sódio (Sigma Aldrich) como fonte de alumínio, sílica gel (Merck) como fonte de silício, $\mathrm{C}_{2} \mathrm{DN}$ (Merck) como direcionador de estrutura, e água
Tabela I - Composição das amostras. [Table I - Composition of the samples.]

\begin{tabular}{ccc}
\hline \multirow{2}{*}{ Amostra } & $\begin{array}{c}\text { Razão } \\
\mathrm{SiO}_{2} / \mathrm{Al}_{2} \mathrm{O}_{3}(\mathrm{SAR})\end{array}$ & $\mathrm{OH} / \mathrm{SiO}_{2}$ \\
\hline 1 & 20 & 0,20 \\
2 & 20 & 0,25 \\
3 & 20 & 0,30 \\
4 & 20 & 0,35 \\
5 & 20 & 0,40 \\
6 & 30 & 0,20 \\
7 & 30 & 0,25 \\
8 & 30 & 0,30 \\
9 & 30 & 0,35 \\
10 & 30 & 0,40 \\
11 & 40 & 0,20 \\
12 & 40 & 0,25 \\
13 & 40 & 0,30 \\
14 & 40 & 0,30 \\
15 & 40 & 0,40 \\
16 & 40 & 0,45 \\
\hline
\end{tabular}

destilada como solvente. O procedimento de síntese ocorreu nas seguintes etapas: dissolução sob agitação do aluminato de sódio e hidróxido de sódio em parte da água, dissolução do $\mathrm{C}_{2} \mathrm{DN}$ (quando utilizado) no restante da água requerida na síntese, adição da fonte de sílica na solução do item (II), seguida de agitação, misturas das soluções (I) e (III), seguida de agitação até completa homogeneização. Após a preparação do gel, este foi transferido para um vaso de teflon revestido com uma autoclave de aço inoxidável e aquecido a $170{ }^{\circ} \mathrm{C}$ por até $240 \mathrm{~h}$. Após a cristalização, a autoclave foi retirada da estufa e resfriada até temperatura ambiente. O seu conteúdo foi transferido para um béquer contendo $100 \mathrm{~mL}$ de água destilada, sendo em seguida submetido a um banho de ultrassom por $25 \mathrm{~min}$ para homogeneização das partículas. Finalmente, o sólido resultante do processo de cristalização foi separado do líquido sobrenadante por filtração a vácuo, lavado diversas vezes com água destilada (até o pH do filtrado atingir o valor próximo a 7) e seco em estufa a $100^{\circ} \mathrm{C}$ por $12 \mathrm{~h}$.

As amostras contendo proporções variadas das fases zeólitas mordenita, ZSM-5 e ferrierita foram selecionadas para validar o método de quantificação de fases desenvolvido neste trabalho.

\section{Análises por difração de raios $X$}

Todas as amostras foram submetidas à caracterização por DRX num equipamento Shimadzu XRD-6000, com radiação $\operatorname{Cuk} \alpha(\lambda=0,1542 \mathrm{~nm})$ com voltagem $30 \mathrm{kV}$ e corrente $30 \mathrm{~mA}$, com filtro de Ni. Os dados foram coletados na faixa de $2 \theta$ de 3 a $50^{\circ}$, com velocidade de varredura do goniômetro de $2^{\circ} \mathrm{min}^{-1}$ com passo $0,02^{\circ}$. 


\section{RESULTADOS E DISCUSSÃO}

\section{Análises térmicas (TG/ATD)}

Foram determinados os percentuais de água intracristalina de cada padrão utilizado para preparação das misturas, sendo determinada uma perda de massa de $7,44 \%$ para ferrierita, $8,10 \%$ para ZSM-5 e $12,61 \%$ para mordenita. Pode-se então realizar-se as correções dos cálculos das massas de cada fase nas misturas.

\section{Análises por difração de raios $X$}

Os difratogramas de raios $\mathrm{X}$ obtidos de cada material foram comparados qualitativamente com os seus padrões obtidos na coleção de DRX simulados para zeólitas [13] e com arquivos PDF (FER 44-0104, MFI 42-0023 e MOR 701883) [14], os quais indicaram a formação das fases (FER,

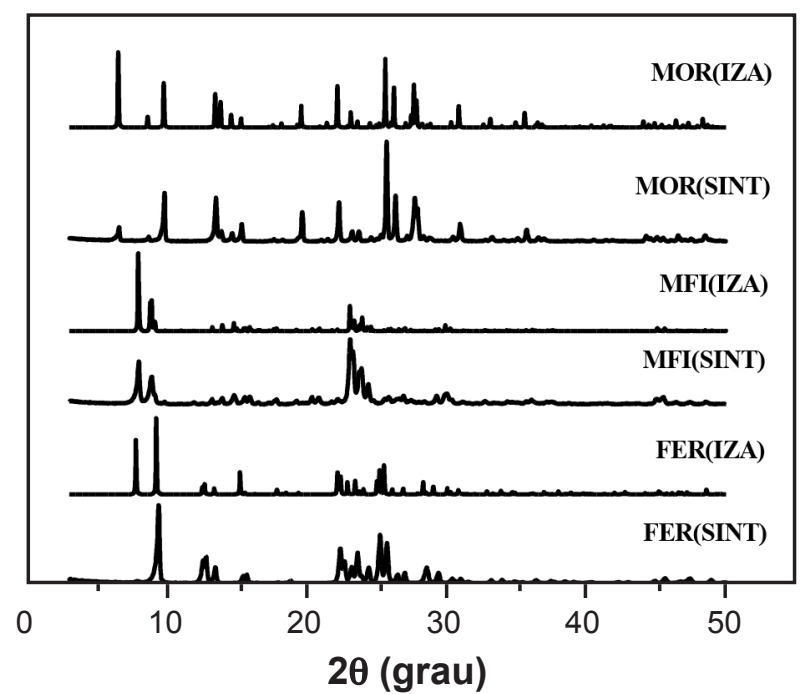

Figura 1: Difratogramas de raios $\mathrm{X}$ das zeólitas sintetizadas em comparação aos padrões obtidos na International Zeolite Association.

[Figure 1: X ray diffraction patterns of the synthesized zeolites compared to patterns obtained from the International Zeolite Association.]

MOR e MFI) em sua forma cristalina pura (Fig. 1).

Posteriormente, foi feita a sobreposição dos difratogramas das estruturas em pares FER-MOR e FER-MFI, onde foram escolhidos os picos característicos de cada material que não se sobrepusesse por nenhum pico do outro componente da mistura (Fig. 2). Dessa forma, tem-se para a zeólita ferrierita o pico na faixa de $2 \theta$ de 24,88 a $25,44^{\circ}$, enquanto que para a mordenita o pico se encontra entre 27,32 e $28,04^{\circ}$ e para a ZSM-5 entre 20,08 e $21,10^{\circ}$.

A partir da identificação dos picos característicos de cada material, foram feitos os cálculos das áreas dos respectivos picos, descontando-se a área referente ao background (radiações oriundas de espalhamentos não coerentes, sem interesse analítico) [15], aplicando-se uma linha de base. As Tabelas II e III apresentam os valores das áreas corrigidas nas misturas FER/MFI do pico correspondente a MFI (Tabela II) e do pico correspondente a FER (Tabela III).

A partir dos valores das áreas obtidas e corrigidas pela subtração da área do background, foram plotados os valores dos teores percentuais de cada fase versus áreas calculadas. A partir destes gráficos foi possível a obtenção de uma relação linear entre o percentual em massa de MFI e FER (Fig. 3) em função da área do pico de cada componente na mistura.

Com base no gráfico entre os teores de MFI e FER com as áreas dos seus picos característicos, realizou-se então uma regressão linear, resultando na equação $\mathrm{y}=0,6747 \mathrm{x}-$ 11,108 e $\mathrm{R}^{2}=0,9989(\mathrm{MFI})$ e $\mathrm{y}=0,1687 \mathrm{x}+3,9819$ e $\mathrm{R}^{2}=$ 0,9980 (FER), por meio das quais pode-se estimar os teores de MFI e FER nas misturas FER/MFI, onde estes foram comparados com os teores reais, sendo então calculados os erros por meio da equação B:

$$
\text { Erro }(\%)=\left(\frac{\text { Teor }_{\text {Real }}-\text { Teor }_{\text {Modelo }}}{\text { Teor }_{\text {Modelo }}}\right) \times 100
$$

Através da Fig. 4, os erros obtidos pela metodologia mostraram comportamento satisfatório para ambas as fases, onde um erro satisfatório se dá em torno de $\pm 5 \%$ [14], ficando evidenciada uma boa aproximação entre os valores dos teores reais e os calculados pelo modelo, obtendo-se um erro na faixa de $\pm 5,06 \%$ para a fase MFI e $\pm 5,53 \%$ para a FER.

Nas Tabelas IV e V são apresentados os valores das áreas corrigidas nas misturas FER/MOR do pico correspondente a MOR (Tabela IV) e do pico correspondente a FER (Tabela $\mathrm{V})$.

A partir dos valores das áreas obtidas e corrigidas pela subtração da área do background, foram então plotados os valores dos teores de cada fase versus áreas calculadas, onde a partir dos gráficos foi possível a obtenção de uma relação linear entre o teor da mordenita e ferrierita (Fig. 5) por área do pico de cada componente da mistura.

Com base no gráfico entre os teores de MOR e FER com as áreas dos seus picos característicos, realizou-se então uma regressão linear, resultando na equação $\mathrm{y}=0,1165 \mathrm{x}+$ 5,4188 e $\mathrm{R}^{2}=0,9963(\mathrm{MOR})$ e $\mathrm{y}=0,1906 \mathrm{x}-10,516$ e $\mathrm{R}^{2}$ $=0,9981$ (FER), através das quais pode-se estimar os teores de cada fase nas misturas FER/MOR, onde estes foram comparados com os teores reais, sendo então calculados os erros (equação B) que são mostrados na Fig. 6.

Verificou-se que de forma similar ao obtido para as misturas FER/MFI, a metodologia apresentou um comportamento similar para os erros obtidos para as misturas FER/MOR, evidenciado uma boa aproximação entre os valores dos teores reais e os calculados pelo modelo, obtendo-se um erro na faixa de $\pm 5,88 \%$ para a fase MOR e $\pm 4,90 \%$ para a FER.

\section{Análise quantitativa das amostras sintetizadas}

As amostras foram sintetizadas com variações de diversos parâmetros de síntese como: composição do 

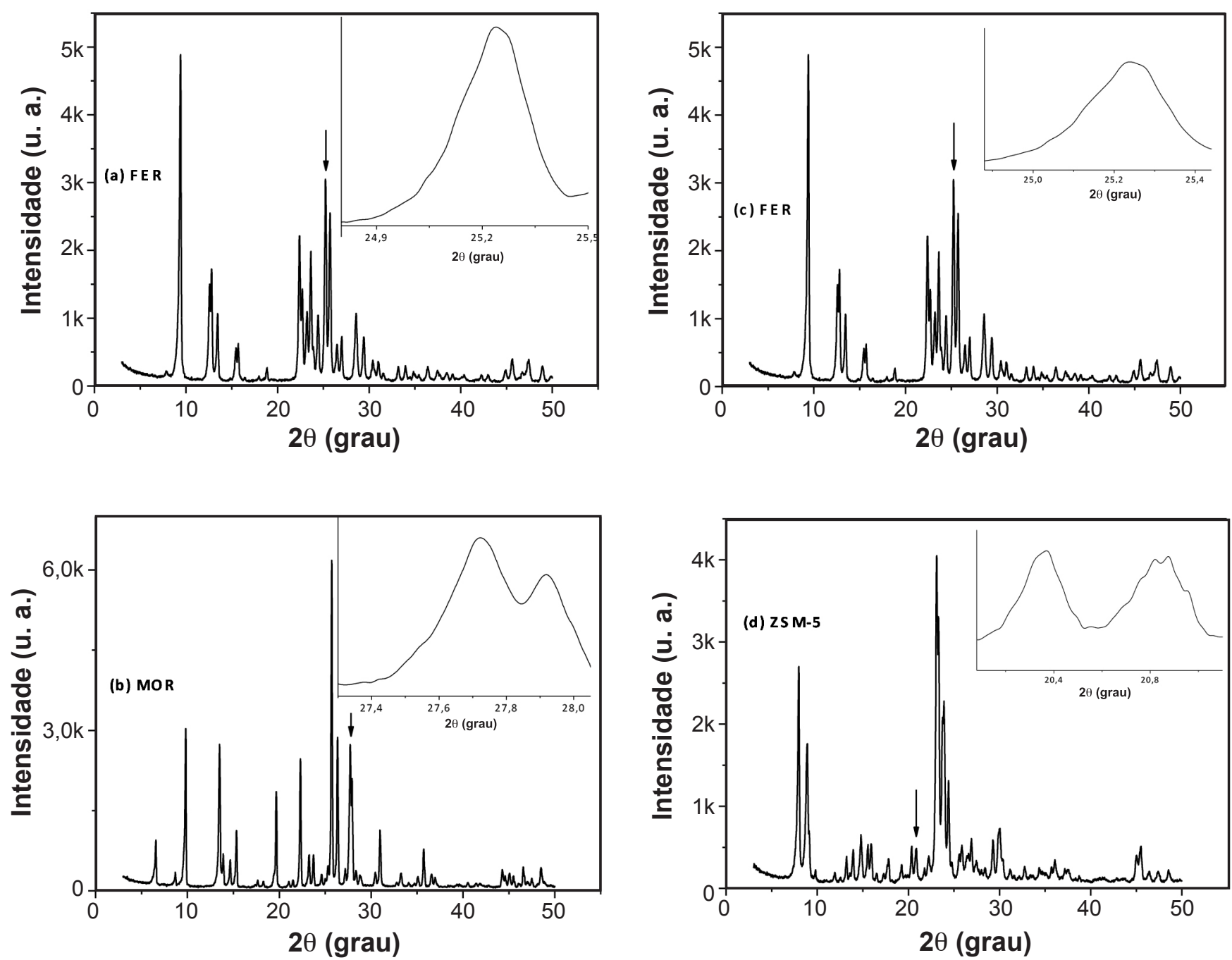

Figura 2: Picos selecionados nas misturas FER/MOR: (a) FER $\left(24,88-25,44^{\circ}\right)$, (b) MOR $\left(27,32-28,04^{\circ}\right)$, e nas misturas FER/MFI: (c) FER $\left(24,88-25,44^{\circ}\right),(d)$ ZSM-5 $\left(20,08-21,10^{\circ}\right)$.

[Figure 2: Selected peaks in mixtures FER/MOR: (a) FER (24.88 to 25.44 $\left.{ }^{\circ}\right)$, (b) MOR (27.32 to 28.04'), and the mixtures FER/MFI: (c) FER (24.88 to $\left.25.44^{\circ}\right)$, (d) ZSM-5 (20.08 to $\left.21.10^{\circ}\right)$.]

gel, teor de direcionador, tempo e temperatura, para a obtenção de amostras que possuíssem a presença de fases binárias (FER/MFI ou FER/MOR) e ternárias (FER/MOR/ MFI). A partir de uma análise qualitativa prévia, através da qual sobrepusemos os padrões de DRX de cada fase sob os difratogramas de cada amostra sintetizada, para a identificação das fases presentes em cada material (Fig. 7).

Através de uma análise qualitativa dos padrões de difração de raios $\mathrm{X}$ das amostras sintetizadas no trabalho em comparação com as amostras contendo as fases puras de mordenita, ZSM-5 e ferrierita, verificou-se que os materiais sintetizados com misturas reacionais com $\mathrm{SAR}=20$ apresentaram uma maior tendência de formação da mistura de fases FER/MOR, já para as de SAR $=30$, observouse uma maior formação de misturas FER/MFI, enquanto as de $\mathrm{SAR}=40$ houve a formação equânime das 3 fases (FER+MOR+MFI).

As áreas dos picos característicos de cada fase na amostra, conforme especificado na Fig. 2, são utilizadas para se estimar o teor da fase. Estes teores são calculados como percentuais pelas relações lineares obtidas com as misturas padronizadas, as quais foram utilizadas $\mathrm{y}=0,6747 \mathrm{x}-11,108$ (MFI) e $\mathrm{y}=0,1165 \mathrm{x}+5,4188$ (MOR). No caso da fase FER tem-se dois modelos disponíveis, um obtido a partir das misturas FER+MOR e outro para as misturas FER+MFI, ambos são bem ajustados a correlação linear, mas o obtido com as misturas FER+MOR ( $\mathrm{y}=0,1906 \mathrm{x}-10,516$ (FER) apresentou um valor de coeficiente de correlação $\mathrm{R}^{2}$ um pouco superior, sendo o modelo escolhido para estimar o teor de ferrierita nas amostras que apresentaram misturas ternárias. Os resultados da aplicação das equações para estimativas de fases nas amostras sintetizadas são mostrados na Tabela VI. Apesar dos modelos terem sido obtidos a partir de misturas padrões binárias de FER+MOR e FER+MFI, quando aplicados a sistemas ternários (amostras 1, 2, 7, 8, 12 e 13), ainda há uma excelente estimativa das concentrações das fases cristalinas presentes. Isto pode ser verificado pelo somatório dos teores de MOR, FER e MFI que variaram de 
Tabela II - Cálculo das áreas para o pico característico da ZSM-5 nas misturas FER/MFI.

[Table II - Calculation of areas of the characteristic peak of ZSM-5 in mixtures FER/MFI.]

\begin{tabular}{ccccc}
\hline Mistura & $\begin{array}{c}\text { Teor de } \\
\text { MFI }\end{array}$ & $\begin{array}{c}\text { Área } \\
\text { Total }\end{array}$ & $\begin{array}{c}\text { Área } \\
\text { Background }\end{array}$ & $\begin{array}{c}\text { Área } \\
\text { Calculada }\end{array}$ \\
\hline 90FERMFI & $10 \%$ & 126,76 & 95,12 & 31,64 \\
85FERMFI & $15 \%$ & 135,50 & 95,70 & 39,80 \\
80FERMFI & $20 \%$ & 142,36 & 95,76 & 46,60 \\
75FERMFI & $25 \%$ & 148,18 & 95,70 & 52,48 \\
70FERMFI & $30 \%$ & 170,68 & 112,00 & 58,68 \\
65FERMFI & $35 \%$ & 173,44 & 103,40 & 70,04 \\
60FERMFI & $40 \%$ & 156,64 & 84,80 & 73,98 \\
55FERMFI & $45 \%$ & 187,52 & 101,92 & 85,60 \\
50FERMFI & $50 \%$ & 204,84 & 114,40 & 90,44 \\
45FERMFI & $55 \%$ & 201,36 & 103,04 & 98,32 \\
40FERMFI & $60 \%$ & 210,80 & 105,84 & 104,96 \\
35FERMFI & $65 \%$ & 231,50 & 119,70 & 111,80 \\
30FERMFI & $70 \%$ & 245,98 & 124,32 & 121,66 \\
25FERMFI & $75 \%$ & 259,74 & 128,82 & 130,92 \\
20FERMFI & $80 \%$ & 257,90 & 121,00 & 136,90 \\
15FERMFI & $85 \%$ & 267,28 & 124,44 & 142,84 \\
10FERMFI & $90 \%$ & 273,28 & 125,44 & 147,84 \\
5FERMFI & $95 \%$ & 289,82 & 133,62 & 156,20 \\
MFI_SINT & $100 \%$ & 303,84 & 138,72 & 165,12 \\
\hline
\end{tabular}

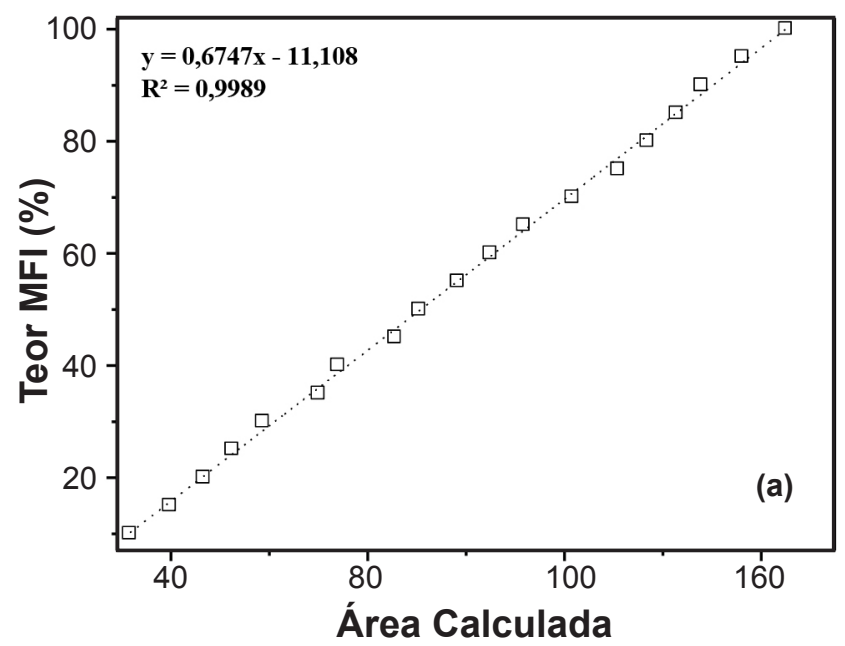

Tabela III - Cálculo das áreas para o pico característico da ferrierita nas misturas FER/MFI.

[Table III - Calculation of areas for the characteristic peak of ferrierite in mixtures FER/MFI.]

\begin{tabular}{ccccc}
\hline Mistura & $\begin{array}{c}\text { Teor de } \\
\text { FER }\end{array}$ & $\begin{array}{c}\text { Área } \\
\text { Total }\end{array}$ & $\begin{array}{c}\text { Área } \\
\text { Background }\end{array}$ & $\begin{array}{c}\text { Área } \\
\text { Calculada }\end{array}$ \\
\hline FER_SINT & $100 \%$ & 811,10 & 232,40 & 578,70 \\
95FERMFI & $95 \%$ & 792,96 & 263,20 & 529,76 \\
90FERMFI & $90 \%$ & 749,02 & 239,12 & 509,90 \\
85FERMFI & $85 \%$ & 734,06 & 262,64 & 471,42 \\
80FERMFI & $80 \%$ & 670,40 & 230,72 & 439,68 \\
75FERMFI & $75 \%$ & 613,48 & 200,48 & 413,00 \\
70FERMFI & $70 \%$ & 595,46 & 188,16 & 407,30 \\
65FERMFI & $65 \%$ & 568,64 & 190,96 & 377,68 \\
60FERMFI & $60 \%$ & 488,70 & 189,10 & 321,40 \\
55FERMFI & $55 \%$ & 488,20 & 180,32 & 307,88 \\
50FERMFI & $50 \%$ & 462,28 & 181,44 & 280,84 \\
45FERMFI & $45 \%$ & 442,86 & 212,80 & 230,06 \\
40FERMFI & $40 \%$ & 374,46 & 154,98 & 219,48 \\
35FERMFI & $35 \%$ & 351,26 & 165,60 & 185,66 \\
30FERMFI & $30 \%$ & 285,76 & 136,32 & 149,44 \\
25FERMFI & $25 \%$ & 259,94 & 133,92 & 126,02 \\
20FERMFI & $20 \%$ & 197,42 & 109,02 & 88,40 \\
15FERMFI & $15 \%$ & 169,64 & 106,72 & 62,92 \\
10FERMFI & $10 \%$ & 135,38 & 99,54 & 35,84 \\
\hline
\end{tabular}

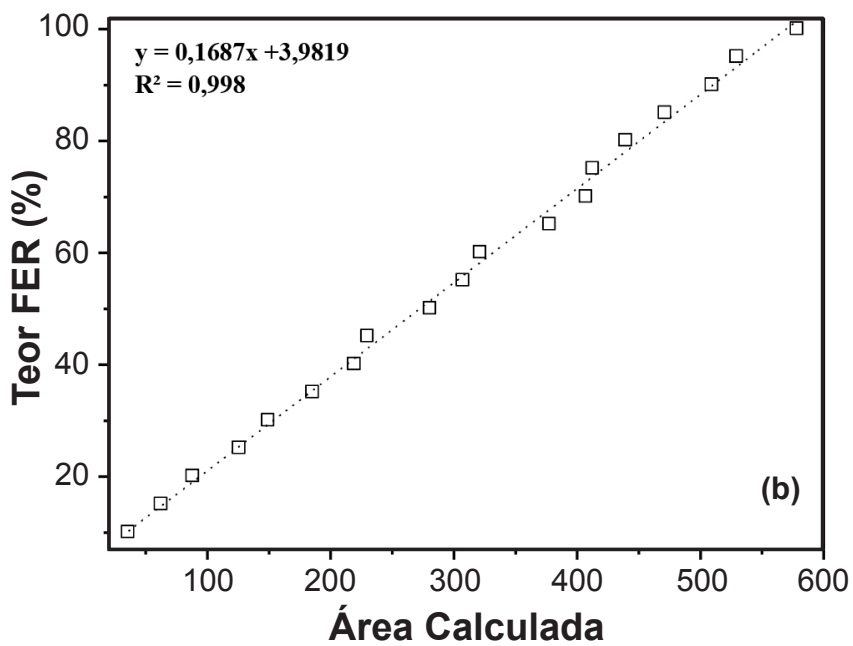

Figura 3: Relação entre os teores de MFI e FER por áreas calculadas dos respectivos picos característicos nas misturas FER/MFI. [Figure 3: Relationship between the levels of MFI and FER by calculated areas of their characteristic peaks in the mixtures FER/MFI.]

97 a $104 \%$, portanto com um erro inferior a $\pm 5 \%$. Estimativas desta ordem de precisão eram alcançadas apenas através de métodos de análises de perfil completo, tais como Rietveld, a partir de um refinamento, muitas vezes, bastante elaborado e demorado [16]. No caso de amostras contendo misturas binárias as estimativas de teores de fases também indicaram valores de somatórios das fases próximos a 100 $5 \%$, o que indica que o método também produz resultados com excelente precisão para amostras sintetizadas contendo misturas das fases FER+MOR e FER+MFI. É importante 

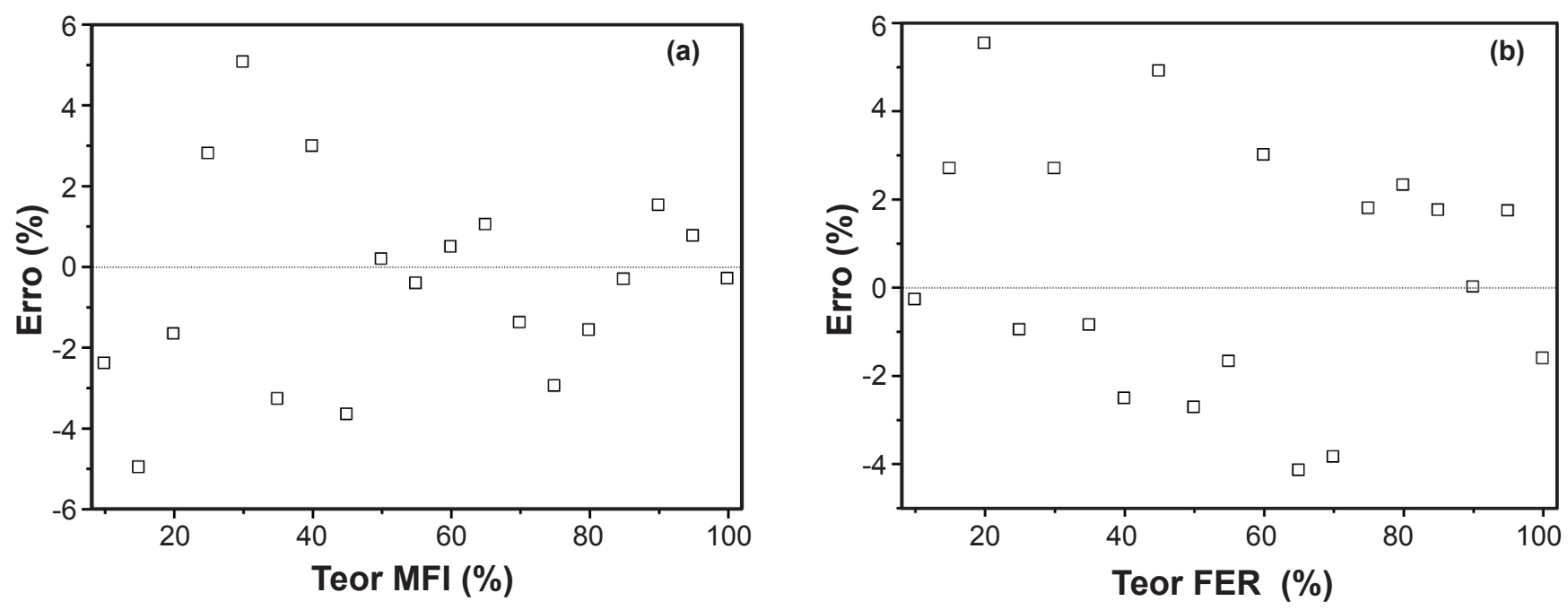

Figura 4: Relação entre os erros e teores de MFI e FER nas misturas FER/MFI.

[Figure 4: Relationship between errors and levels of MFI and FER in mixtures FER /MFI.]

Tabela IV - Cálculo das áreas para o pico característico da mordenita nas misturas FER/MOR.

[Table IV - Calculation of areas for the characteristic peak of mordenite in mixtures FER/MOR.]

\begin{tabular}{ccccc}
\hline Mistura & $\begin{array}{c}\text { Teor de } \\
\text { MOR }\end{array}$ & $\begin{array}{c}\text { Área } \\
\text { Total }\end{array}$ & $\begin{array}{c}\text { Área } \\
\text { Background }\end{array}$ & $\begin{array}{c}\text { Área } \\
\text { Calculada }\end{array}$ \\
\hline 90FERMOR & $10 \%$ & 128,12 & 85,84 & 42,28 \\
85FERMOR & $15 \%$ & 182,68 & 100,80 & 81,88 \\
80FERMOR & $20 \%$ & 228,76 & 103,60 & 125,16 \\
75FERMOR & $25 \%$ & 269,70 & 101,38 & 168,32 \\
70FERMOR & $30 \%$ & 334,92 & 111,52 & 223,40 \\
65FERMOR & $35 \%$ & 354,70 & 118,44 & 236,26 \\
60FERMOR & $40 \%$ & 414,92 & 116,64 & 298,28 \\
55FERMOR & $45 \%$ & 463,84 & 122,40 & 341,44 \\
50FERMOR & $50 \%$ & 507,08 & 116,92 & 390,16 \\
45FERMOR & $55 \%$ & 542,32 & 125,28 & 417,04 \\
40FERMOR & $60 \%$ & 572,36 & 128,16 & 444,20 \\
35FERMOR & $65 \%$ & 668,26 & 133,20 & 535,06 \\
30FERMOR & $70 \%$ & 686,54 & 138,96 & 547,58 \\
25FERMOR & $75 \%$ & 729,72 & 132,60 & 597,12 \\
20FERMOR & $80 \%$ & 800,60 & 145,08 & 655,52 \\
15FERMOR & $85 \%$ & 818,00 & 143,56 & 674,44 \\
10FERMOR & $90 \%$ & 870,06 & 171,50 & 698,56 \\
5FERMOR & $95 \%$ & 877,58 & 145,16 & 732,42 \\
MOR_SINT & $100 \%$ & 1000,92 & 160,72 & 840,20 \\
\hline
\end{tabular}

destacar que a aplicação desta metodologia rotineiramente pode requerer que periodicamente as curvas de calibração
Tabela V - Cálculo das áreas para o pico característico da ferrierita nas misturas FER/MOR.

[Table V-Calculation of areas for the characteristic peak of ferrierite in mixtures FER/MOR.]

\begin{tabular}{ccccc}
\hline \multirow{2}{*}{ Mistura } & $\begin{array}{c}\text { Teor de } \\
\text { FER }\end{array}$ & $\begin{array}{c}\text { Área } \\
\text { Total }\end{array}$ & $\begin{array}{c}\text { Área } \\
\text { Background }\end{array}$ & $\begin{array}{c}\text { Área } \\
\text { Calculada }\end{array}$ \\
\hline FER_SINT & $100 \%$ & 811,10 & 232,40 & 578,70 \\
95FERMOR & $95 \%$ & 771,00 & 222,88 & 548,12 \\
90FERMOR & $90 \%$ & 742,48 & 222,88 & 519,60 \\
85FERMOR & $85 \%$ & 707,92 & 217,28 & 490,64 \\
80FERMOR & $80 \%$ & 698,50 & 214,48 & 484,02 \\
75FERMOR & $75 \%$ & 679,04 & 226,24 & 452,80 \\
70FERMOR & $70 \%$ & 637,32 & 209,44 & 427,88 \\
65FERMOR & $65 \%$ & 619,60 & 210,08 & 409,52 \\
60FERMOR & $60 \%$ & 608,08 & 242,40 & 365,68 \\
55FERMOR & $55 \%$ & 549,40 & 200,48 & 348,92 \\
50FERMOR & $50 \%$ & 520,80 & 207,20 & 313,60 \\
45FERMOR & $45 \%$ & 503,32 & 200,48 & 302,84 \\
40FERMOR & $40 \%$ & 482,90 & 211,12 & 271,78 \\
35FERMOR & $35 \%$ & 419,44 & 175,84 & 243,60 \\
30FERMOR & $30 \%$ & 393,78 & 186,48 & 207,30 \\
25FERMOR & $25 \%$ & 365,80 & 175,84 & 189,96 \\
20FERMOR & $20 \%$ & 332,96 & 173,60 & 159,36 \\
15FERMOR & $15 \%$ & 283,30 & 152,88 & 130,42 \\
10FERMOR & $10 \%$ & 249,78 & 139,86 & 109,92 \\
\hline
\end{tabular}

sejam reajustadas para compensar as variações de potência na intensidade do sinal causada pelo uso continuado do 

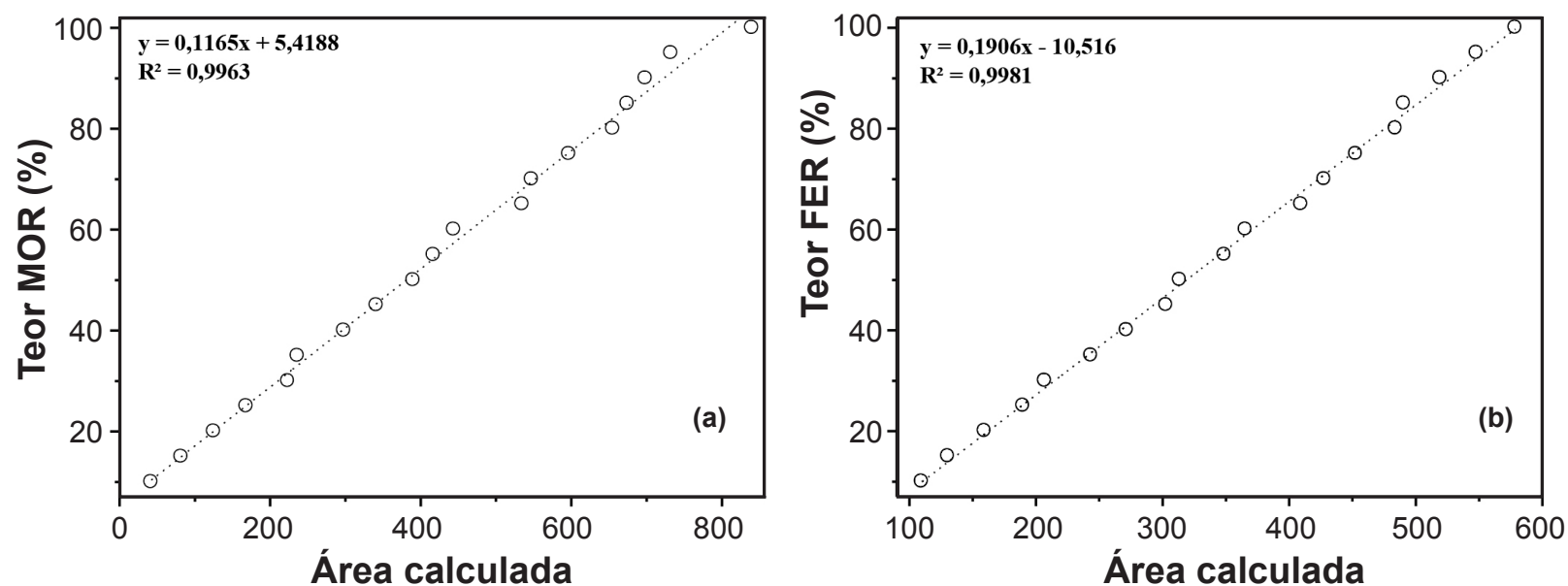

Figura 5: Relação entre os teores de MOR e FER por áreas calculadas dos respectivos picos característicos nas misturas FER/MOR. [Figure 5: Relationship between the levels of MOR and FER by calculated areas their characteristic peaks in the mixtures FER/MOR.]
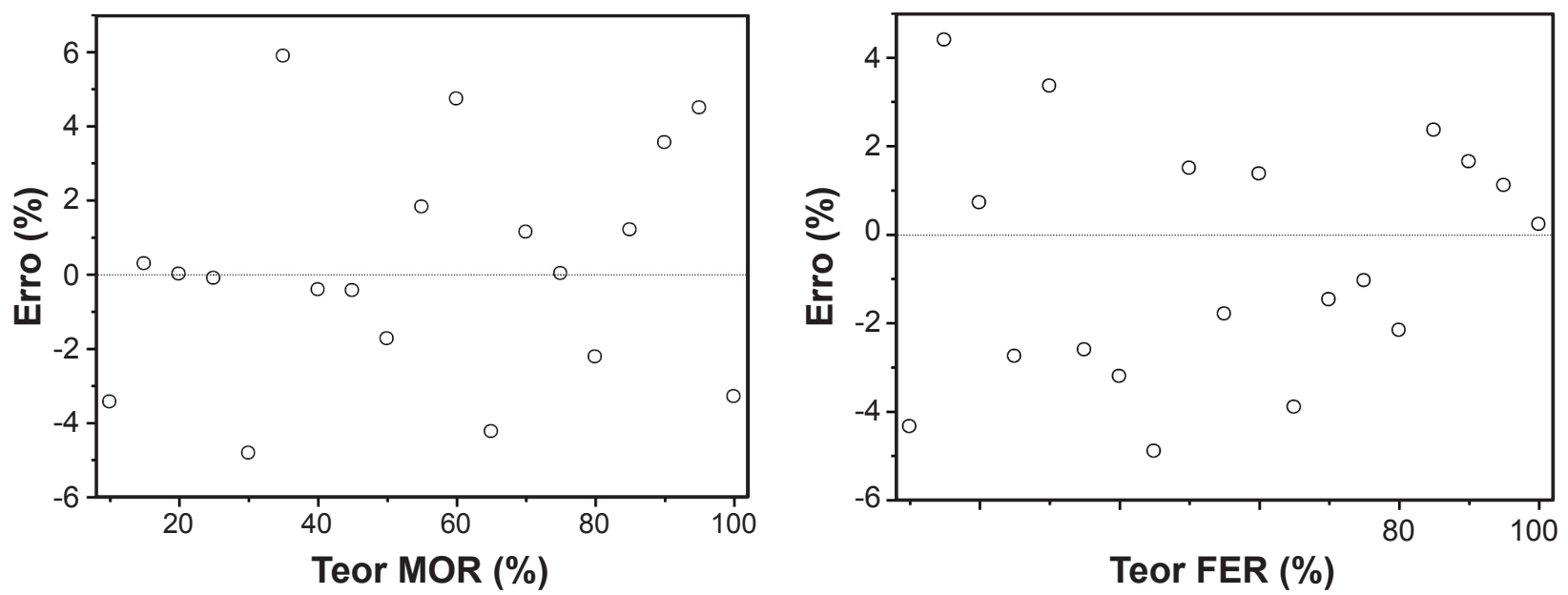

Figura 6: Relação entre os erros e teores de MOR e FER nas misturas FER/MFI.

[Figure 6: Relationship between errors and levels of MOR and FER in mixtures FER /MOR.]

Tabela VI - Quantificação das fases nas amostras sintetizadas.

[Table VI - Quantification of the phases in the synthesized samples.]

\begin{tabular}{ccccc}
\hline Amostra & $\begin{array}{c}\text { Teor de MOR } \\
(\%)\end{array}$ & $\begin{array}{c}\text { Teor de FER } \\
(\%)\end{array}$ & $\begin{array}{c}\text { Teor MFI } \\
(\%)\end{array}$ & $\begin{array}{c}\text { Total } \\
(\%)\end{array}$ \\
\hline 1 & 44 & 28 & 26 & 98 \\
2 & 30 & 57 & 9 & 97 \\
3 & 91 & 8 & 0 & 98 \\
4 & 57 & 42 & 0 & 99 \\
5 & 32 & 67 & 0 & 99 \\
6 & 0 & 32 & 70 & 101 \\
7 & 4 & 45 & 52 & 101 \\
8 & 4 & 47 & 46 & 97 \\
9 & 35 & 69 & 0 & 103 \\
10 & 22 & 84 & 0 & 105 \\
11 & 0 & 46 & 57 & 103 \\
12 & 13 & 38 & 54 & 104 \\
13 & 6 & 77 & 15 & 98 \\
14 & 37 & 63 & 0 & 101 \\
15 & 62 & 34 & 0 & 96 \\
16 & 0 & 31 & 68 & 98 \\
\hline
\end{tabular}

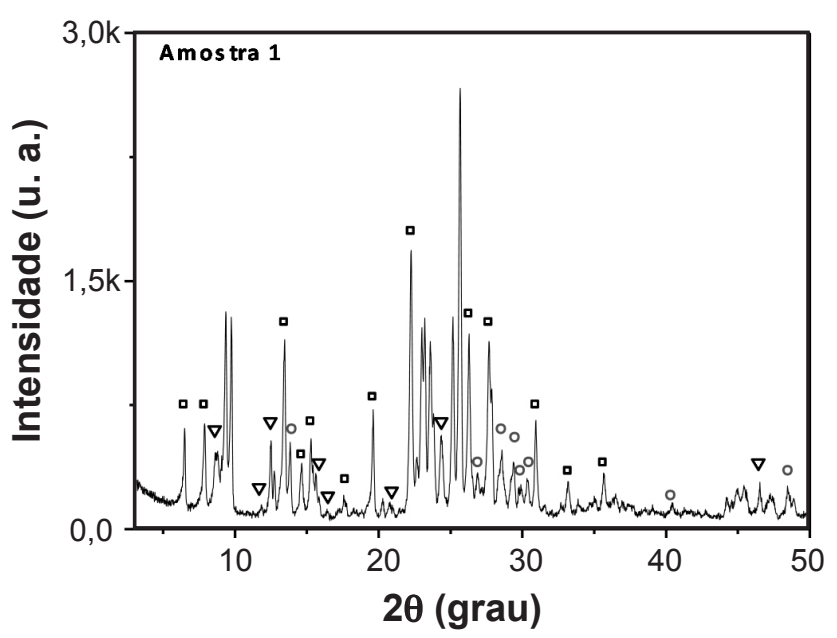

Figura 7: Difratograma de raios X das amostras sintetizadas (MOR $\square$, MFI $\nabla$ e FERO), picos não sinalizados são sobreposição de fases).

[Figure 7: X-ray diffraction of the synthesized samples (MOR $\square$, $M F I \nabla$ and FERO), unmarked peaks are overlapping phases.] 
tubo de raios $\mathrm{X}$, ou mesmo quando são feitos ajustes no equipamento.

A Tabela VI apresenta os resultados obtidos na quantificação das fases presentes nas amostras sintetizadas.

\section{CONCLUSÕES}

Asíntese de zeólitas em diferentes condições experimentais produziu materiais com concentrações variadas das fases FER, MOR e MFI. De forma a estimar o percentual de fases presentes nas amostras foram desenvolvidos modelos a partir de misturas binárias de ferrierita e das fases contaminantes. $\mathrm{O}$ emprego destas metodologias experimentais permitiu obter as seguintes conclusões: os modelos binários, contendo misturas de fases FER+MOR e FER+MFI em diferentes concentrações, apresentaram uma excelente correlação linear, com coeficientes de ajustes $\left(R^{2}\right)$ superior a 0,9960 ; a distribuição dos erros entre os valores reais do percentual de fases nos padrões e os estimados pelos modelos mostraram uma distribuição aleatória em função da concentração real, sendo um indicativo da excelente qualidade do procedimento adotado; o erro percentual para estimativas das fases nas amostras padrões a partir dos modelos obtidos foram da ordem de $\pm 6 \%$; a síntese de zeólita ferrierita nas diversas condições experimentais estudadas, indicaram que a contaminação pela fase mordenita é favorecida em SAR baixo, enquanto que a fase contaminante ZSM-5 é mais comum em SAR intermediários. No caso de $\mathrm{SAR}=40$ há uma maior tendência a formação de misturas ternárias das fases FER, MOR e MFI. A aplicação dos modelos obtidos no trabalho para estimativas de teor de fases nas amostras sintetizadas resultou em excelentes estimativas das concentrações das fases cristalinas, conforme pode ser verificado pelo somatório dos teores de MOR, FER e MFI que variaram de 97 a 104\% nas amostras formadas por misturas ternárias. Isto implica num erro percentual inferior a $\pm 5 \%$. No caso de amostras contendo misturas binárias as estimativas de teores de fases também indicaram valores de somatórios das fases próximos a $100 \pm 5 \%$, o que indica que o método também produz resultados com excelente precisão nestes casos.

\section{REFERÊNCIAS}

[1] J. Weitkamp, Solid State Ionics 131 (2000) 175.

[2] E. M. Flanigen, Studies Surface Sci. Catalysis 137 (2001)13.

[3] A. P. F. Albers, F. G. Melchiades, R. Machado, J. B. Baldo, A. O. Boschi, Cerâmica 48, 305 (2002) 305.

[4] G. Perego, Catalysis Today 41 (1998) 251.

[5] R. Szostak, Molecular Sieves: Principles of Synthesis and Identification, $2^{\text {nd }}$ Ed., Blackie Academic \& Professional, New York, EUA (1998) 283.

[6] L. V. Sousa Júnior, A. O. S. Silva, B. J. B. Silva, S. L. Alencar, Modern Res. Catalysis 3 (2014) 51.

[7] T. Wessels, Ch. Baerlocher, L. B. McCusker, E. J. Creyghton, J. Am. Chem. Soc. 121 (1999) 6242.

[8] F. R. Ribeiro, J. L. Figueiredo, Catálise Heterogênea, $2^{\mathrm{a}}$ ed., Fundação Calouste Gulbenkian, Lisboa, Portugal (2007) 25.

[9] C. Baerlocher, L. B. McCusker, Studies Surface Sci. Catalysis 85 (1994) 391.

[10] A. W. Chester, E. G. Derouane, "Zeolite Characterization and Catalysis", Springer. New York, EUA (2009) 1-2.

[11] P. A. Jacobs, J. A. Martens, Studies Surface Sci. Catalysis 33 (1987) 217.

[12] A. O. S. Silva, "Sintese e Caracterização de Catalisadores de Ferro e Cobalto Suportados nas Zeólitas HZSM-12 e HZSM-5 para a Conversão de Gás de Síntese em Hidrocarbonetos", Tese Dr. Eng. Quim., Universidade Federal do Rio Grande do Norte, Natal, RN (2004) p.38.

[13] M. M. J. Treacy, J. B. Higgins, R von Ballmoos, "Collection of Simulated XRD Powder Patterns for Zeolites," Zeolites 16 (1996).

[14] PDF Database (Sets 1-44), International Centre for Diffraction Data, EUA (1994).

[15] J. R. Connolly, "Introduction Quantitative X-ray Diffraction Methods" (2013), http://epswww.unm.edu/xrd/ xrdclass/09-Quant-intro.pdf

[16] R. D. Bonetto, P. E. Zalba, M. S. Conconi, M. Manassero, Rev. Geol. Chile 30 (2003) 113-115.

(rec. 11/06/2014, Rev. 17/09/2014, Ac. 31/10/2014) 